\title{
Humanização em situações de abortamento: relato de experiência em uma maternidade pública
}

\author{
Humanization in abortion situations: report of experience in public maternity
}

Humanización en situación del aborto: relato de la experiencia en una maternidad publica

Gabriella Furtado Monteiro ${ }^{1 *}$, Ádria Sthella Guedes Paladino1, Darci Francisco dos Santos Junior ${ }^{1}$, Larissa Leite Pelaes ${ }^{1}$, Leilson da Silva Lima1, Luiza Picanço Nunes ${ }^{1}$, Nádia Cecília Barros Tostes", Vanessa da Silva Oliveira'.

\section{RESUMO}

Objetivo: Relatar a experiência diante de um caso de um natimorto no ambiente hospitalar e discutir a humanização. Trata-se de um relato de experiência, vivenciado por alunos de graduação do oitavo semestre do curso de Bacharelado em Enfermagem, durante a disciplina "Estágio Supervisionado II": área hospitalar em maternidade de referência no Estado do Amapá. Relato de experiência: Durante a prática do estágio supervisionado em uma maternidade de referência no Estado do Amapá, observou-se na enfermaria de atendimento pós-parto imediato, que várias mulheres dividiam leitos com outras mães e seus recém-nascidos, pois havia leitos e berços em quantidade suficiente para acomodação adequada, devido ao alto índice de ocupação hospitalar. No contexto do ambiente apresentado, uma gestante em período expulsivo iniciou o protocolo de indução medicamentosa para o parto de um filho natimorto. Considerações finais: A vivência e a discussão com a literatura científica reforçam que a prerrogativa da assistência prestada à mulher em óbito fetal deve ser um processo contínuo de educação em saúde, tanto nas universidades quanto nos setores de trabalho dos profissionais de saúde.

Palavras-chave: Humanização da assistência, Natimorto, Enfermagem materno-Infantil.

\begin{abstract}
Objective: Report the experience up to a case of a stillborn in the hospital environment and discuss about the humanization. Experience report: In the attendance infirmary immediate postpartum, was observed that a lot of women share their beds with other mothers and their newborns, because there was no beds and cribs in sufficient quantity to a suitable accommodation, due to due to the high rate of hospital occupancy. In the context of the presented environment, a pregnant in the expulsive period initiate the protocol of drug induction to childbirth of a stillborn child. The observed problems by the authors, the hospital infrastructure in particular, had directly reflex in the assistance humanization, although the team that provided assistance to the pregnant has adopted measures aimed at the patient's privacy and respect in abort conditions. Final considerations: The experience and discussion with the scientific literature reinforces the prerogative of the assistance provided to women with fetal death should be a continuous process of health education, both within the universities and in the work sectors of health professionals.
\end{abstract}

Key words: Humanization of assistance, Stillbirth, Maternal-child nursing.

\section{RESUMEN}

Objetivo: Relatar la experiencia frente a un caso de un nascido muerto en el ámbito hospitalario y discutir la humanización. Es de un relato de experiencia, vivida por estudiantes de graduación del octavo semestre de la carrera de Enfermería, durante la disciplina de pasantía supervisada II: Área hospitalaria en la maternidad de referencia en el Estado de Amapá. Relato de experiencia: Durante la pratica de pasantía supervisionada en una maternidad de referencia en el Estado de Amapá, se observó en la sala de atención de posparto inmediato, que varias mujeres compartían cama con otras madres y sus recién nacidos, ya que no había

${ }^{1}$ Universidade Federal do Amapá (UNIFAP), Macapá - AP. *E-mail: gabifurtado.97@gmail.com

PUBLICADO EM: 3/2021 
suficientes camas y cunas para acomodarlas adecuadamente, debido a la gran tasa de ocupación hospitalaria. En el contexto del ambiente presentado, una mujer embarazada en el período expulsivo inició el protocolo de inducción de fármacos para el parto de un niño Nacido muerto. Consideraciones finales: La experiencia y discusión con la literatura científica refuerza la prerrogativa de que la asistencia brindada a las mujeres con muerte fetal debe ser un proceso continuo de educación en salud, tanto en las universidades como en los sectores laborales de los profesionales de la salud.

Palabras clave: Humanización de la atención, Nascido muerto, Enfermería materno-infantil.

\section{INTRODUÇÃO}

A saúde da mulher e da criança é um assunto de relevância global, como destaca a Organização das Nações Unidas (ONU) que criou no ano 2000 como meta do desenvolvimento do Milênio até 2015, a redução em dois terços da mortalidade de crianças menores de cinco anos de idade e melhora da saúde materna, porém esses indicadores não foram atingidos (CARLLO WA e TRAVERS CP, 2016).

No Brasil, o Ministério da Saúde (MS) instituiu políticas públicas de saúde presentes em todo o território nacional, com o objetivo de reorganizar a rede de atenção à saúde para promover a melhora na qualidade de vida da população. No entanto, apesar dos esforços nas últimas décadas para superar as altas taxas de morbimortalidade materna e infantil, que continuam elevados, sobretudo a neonatal, foi criada a Rede Cegonha que tem como um dos objetivos a redução dessas taxas (BRASIL, 2011).

Muitos obstáculos dificultam a redução da mortalidade materno infantil. Dentre elas está a dificuldade de atentar-se à pluralidade cultural, implantar diretrizes do acolhimento e da clínica ampliada e aprimorar a interação multidisciplinar e qualificá-las para lidarem com as singularidades dos sujeitos e coletividade nas práticas (BRASIL, 2008).

Diante desse contexto, surge a política nacional de humanização que visa a valorização da autonomia e o protagonismo do sujeito, escolhas e singularidades dos indivíduos. Esse processo deve estar presente em toda atenção de saúde, principalmente em áreas que historicamente apresentam desrespeito aos direitos das mulheres (BRASIL, 2010; POSSATI AB, et al., 2017).

Uma das políticas preconizadas pelo MS foi o modelo de atenção humanizado às mulheres em situação de abortamento, com o objetivo de oferecer nova referência de maneira segura, sustentável e sucedida à atenção. Segundo o MS, em todos os casos de atenção deve ser utilizado o apoio empático e humanizado para proporcionar menores riscos de complicação e mais rápida recuperação (BRASIL, 2011).

Dessa forma para que haja a ambiência humanizada há necessidade da criação de espaços saudáveis e acolhedores que permitam a privacidade visual e verbal, englobando tanto tecnologias médicas, estética e componente sensível como olfato e ruídos (BRASIL, 2010).

Além do espaço humanizado, é necessário que os profissionais da saúde tenham conhecimento sobre as políticas e recomendações do MS. Entre os profissionais, os enfermeiros se destacam por estarem próximo da mulher no período de pré-natal, parto e puerpério e por acreditarem na importância do empoderamento das usuárias e no resgate do parto como um evento natural e fisiológico (POSSATI AB, et al., 2017).

Apesar do empoderamento, há o risco de a mãe vivenciar a perda do filho no período perinatal e sentir uma dor que pode ser devastadora. Além disso, poderá afetar gestações futuras, já que é possível que essa situação propicie o surgimento do medo de passar pela mesma intercorrência, ocasionando o distanciamento emocional durante a gravidez, que gera dificuldade de criar laços afetivos com o bebê e até desencadeia a depressão pós-parto (MENDONÇA CSA, 2018; LOPES BG, et al., 2019).

Dessa maneira, os profissionais da saúde devem ser qualificados para assistência da mulher em óbito fetal e implementar ações que possibilitem a humanização desse momento difícil. Diante disso surge a figura da Enfermagem obstétrica, não obstante a esse profissional, mas toda a equipe precisa desenvolver habilidades de comunicação, reflexão e implementação de estratégias de educação permanente com objetivo 
de promover à atenção integral da mulher que vivencia o óbito perinatal (LOPES BG, et al., 2019; LARI LR, et al., 2018; DUARTE MG, 2019).

A humanização e qualidade da atenção implicam em respeito aos direitos humanos garantindo a integralidade da assistência e satisfação (BRASIL, 2004). Tendo como direitos o sigilo profissional e respeito aos princípios da bioética, tal qual a beneficência, não-maleficência, autonomia e a justiça, e a humanização às mulheres em situação de abortamento (BRASIL, 2011).

É preciso centrar no usuário para compreender a humanização do parto e os efeitos positivos. Faz-se necessário também atentar-se aos anseios, dúvidas e queixas para propor mudanças, ou então, perpetuarão cenários caracterizados por estrutura física precária, com atuação focada em técnicas e intervenções orientadas por normas e rotinas (POSSATI AB, et al., 2017). Muitas vezes pode ocorrer a carência de apoio psicológico e medidas que poderiam ser determinantes em uma melhor vivência do processo de luto após a perda gestacional (MENDONÇA CSA, 2018).

Dessa maneira deve ser formado um novo conceito de saúde que promova a consciência da cidadania dos indivíduos à humanização do nascimento, a qual apenas será possível se os profissionais de saúde, gestantes e sociedade em geral, assimilarem esses conceitos (SANTOS HFL e ARAUJO MM, 2016).

Diante desse contexto, o objetivo desse trabalho é relatar a experiência diante de um caso de um natimorto no ambiente hospitalar de um hospitalar de referência no Estado do Amapá e discutir a respeito da humanização.

\section{RELATO DE EXPERIÊNCIA}

Este estudo é caracterizado como relato de experiência vivenciado por graduandos do oitavo semestre do curso de bacharelado em Enfermagem, durante a disciplina Estágio Supervisionado II: área hospitalar na maternidade, referência no Estado do Amapá.

O Estágio Supervisionado II na maternidade possui como atividade integrante a assistência prestada no Centro de Parto Normal (CPN) com trabalho de parto, parto e nascimento e na enfermaria mista, onde ocorre a prestação de cuidados pós-parto imediato. Ao chegar na maternidade, o grupo se dividiu de acordo com suas funções em seus respectivos setores, ficando sob responsabilidade de uma das acadêmicas as atividades prestadas na enfermaria mista em questão.

A enfermaria de cuidados pós-parto imediato é um ambiente pequeno, onde encontravam-se 21 pacientes (em sua maioria puérperas e uma mulher em período expulsivo de um natimorto). Observou-se que várias mulheres dividiam leitos com outras puérperas e seus recém-nascidos, pois não havia leitos e berços em quantidade suficiente para a acomodação adequada no local, devido à alta taxa de ocupação hospitalar e que também havia apenas um biombo na sala.

A mulher em período expulsivo do natimorto que estava presente na enfermaria de cuidados pós-parto havia iniciado o protocolo de indução medicamentosa para o parto durante a madrugada, mas somente durante o período da manhã ela atingiu a dilatação completa para a realização do parto. Devido a lotação e necessidade de realocação da equipe para suprir a demanda das atividades, a acadêmica responsável não estava ciente que a situação era o parto de um natimorto morto há aproximadamente três dias.

Por volta das 08:30 iniciou-se seu parto (natimorto, 28 semanas e com apresentação pélvica) assistido pela médica residente e pela acadêmica, que soube da particularidade do parto durante o momento de expulsão, Devido a lotação da enfermaria, solicitou aos acompanhantes das outras pacientes que se retirassem do local, com objetivo de oferecer maior privacidade. A médica residente precisou realizar as manobras de parto pélvico para a expulsão do concepto.

Ressalta-se ainda que foi realizado o protocolo para evitar hemorragia pós-parto e após a dequitação, a paciente apresentou-se chorosa e abalada com a situação. Em seguida chegou à enfermaria a mãe e o companheiro da paciente solicitando à médica residente que os deixassem ver o natimorto. A paciente ainda foi questionada pela médica se desejava ver o feto e a mulher chorando copiosamente apenas balançou a 
cabeça negando-se a vê-lo. Nesse momento na sala o que predominava era um silêncio absoluto de todos, interrompido apenas pelos sons dos RNs presentes. O natimorto permaneceu enrolado por compressas na sala durante cerca de 20 minutos.

Durante o momento do relato citado é importante ressaltar que nenhuma das acadêmicas presentes tiveram anteriormente em uma vivência parecida, fato que foi perceptível pelos profissionais do hospital, os quais foram compreensivos com os acadêmicos e posteriormente debatido de modo humanizado e ético com o professor, alunos e profissionais sobre a situação e condições na qual ocorreu.

\section{DISCUSSÃO}

O Sistema Único de Saúde (SUS) apresentou mudanças para a implementação da promoção da saúde das gestantes, uma história marcada por avanços em pontos como assistência ao pré-natal e atenção qualificada ao parto, incluindo aspectos como a redução das desigualdades sociais e diferenças regionais. Em um estudo nacional de causas de óbitos neonatais prevaleceu as mortes por prematuridade, respondendo por cerca de $1 / 3$ dos casos, seguidos pela malformação congênita $(22,8 \%)$, as infecções $(18,5 \%)$, os fatores maternos $(10,4 \%)$ e asfixia/hipóxia (7\%). Na região Norte prevaleceu óbitos registrados como infecção, ou seja, o predomínio de causas evitáveis (LEAL MC, et al., 2018; LANSKY, et al., 2014).

Devido isso em 2017 foi instituído na maternidade um projeto de aprimoramento e inovação no cuidado e ensino em obstetrícia e neonatologia, o Apice ON. O Apice ON na Maternidade Pública do Estado foi composto por uma equipe multiprofissional formada por médicos, enfermeiros e técnicos de enfermagem. Para em um período de 2 anos desenvolver as atividades propostas pelo MS. Entre as atividades propostas está a atenção às mulheres em situação de abortamento e aborto legal. Esse projeto se dá na realidade marcada pela permanência de problemas sociais e epidemiológico-sanitário relacionado à atenção obstétrica e neonatal como a mortalidade neonatal e atenção humanizada ao abortamento.

A perda perinatal envolve a equipe multidisciplinar. A prática de cuidados e acompanhamento não deve ser realizada de maneira improvisada, portanto, faz-se necessário desenvolver habilidades especiais sobre a comunicação, ajuda e pesar perinatal, de modo a serem desenvolvidos desde a formação acadêmica (PASTOR SM, 2011).

Apesar de seus avanços em políticas de saúde o SUS tem apresentado sucateamento, desqualificação dos serviços públicos de saúde e a precarização. Esses obstáculos estão presentes desde a formação onde ainda dificuldades no trabalho em equipe multiprofissional e pouco comprometimento com as políticas públicas de saúde, sendo preciso ver a enfermagem como parte da estrutura social que possibilita mudanças que resultam em ações de cidadania, promoção de saúde e redução das tendenciosidades (ARAUJO JL, 2018).

As dificuldades estruturais, a falta de recursos, de equipamentos e de materiais nos hospitais precariza o trabalho dos profissionais e a assistência em saúde. Em geral, as áreas físicas das maternidades não foram construídas para permitir a humanização do cuidado. Esses problemas em conjunto a superlotação de hospitais torna o funcionamento do sistema de saúde deficiente de forma geral, devido a escassez dos recursos em relação à elevada demanda de usuárias, tornando difícil a implementação da assistência humanizada (PARADA CMGL e CARVALHÃES MBL, 2007; O'DWYER GO, et al., 2009; DODOU HD, et al., 2017).

Segundo Lima ML, et al. (2017) as mulheres em situação de abortamento são submetidas a um contínuo processo de violência e desrespeito nas instituições de saúde em situação de abortamento. Dentre as violências institucionais sofridas, Madeiro AP e Rufino AC (2017) destacam o julgamento moral, tratamento não digno, negligências, violação da privacidade e confidencialidade, além dos procedimentos realizados sem explicação e consentimento dos profissionais de saúde.

A assistência humanizada diante ao processo de abortamento cabe aos profissionais de saúde, sobretudo a equipe de enfermagem, devido ao contato contínuo e próximo aos pacientes. 0 compromisso com o acolhimento como mecanismo de cuidado é imprescindível, ofertando palavras de conforto, atenção, 
explicação dos procedimentos, por exemplo, são formas simples de promover essa humanização, diminuindo os medos, angústia e sofrimento (SILVA MJS, et al., 2020; SOARES MCS, et al., 2012).

Naturalmente a morte de um filho causa dor, revolta e trazem sentimentos de incompreensão, principalmente quando este é um filho que ainda não saiu da barriga da mãe. Para Luna IJ e Moré CO (2019) o luto representa e apresenta-se quando há rompimento de vínculos, perda específica de um ente querido ou de algo. Neste caso, o processo de humanização que a enfermagem deve desempenhar é de apoio, buscando escutar e respeitar o paciente e encaminhá-lo para serviços de apoio como assistência social e psicológica.

As mulheres que sofrem uma perda precoce durante a gravidez podem apresentar níveis altos de estresse, ansiedade e depressão. Não obstante, essas problemáticas podem prevalecer durante meses. (FARREN J, et al., 2020). Tais implicações mentais podem ocasionar o desenvolvimento de sinais e sintomas que diminuem a qualidade de vida da mulher, como o sentimento de inutilidade ou culpa excessiva ou inapropriada, fadiga, irritabilidade, tensão muscular, perturbação do sono, aumento de ideação suicida ou até mesmo suicídio. Ademais, prejuízo imensurável no funcionamento social e profissional da grávida (CARVALHO AJ, 2019).

A ausência de cuidados relacionados à ansiedade materna pode afetar o cuidado com os próximos filhos, como exemplo a diminuição do período de amamentação (LEÓN, et al., 2018). Dessa maneira diminuindo os vínculos afetivos saudáveis entre mãe e filho. Não obstante pode trazer prejuízos relacionados a formação orofacial do recém-nascido (MENEZES RR, et al., 2019)

Dessa maneira é de suma importância a necessidade de instrumentos que avaliem sistematicamente a ansiedade e depressão na gestação. Tais alterações psicológicas não são influenciadas só pelas incertezas do período gravídico, mas por diversos fatores e por sua identificação. Diante disso, estar atento à essas alterações é de suma importância para o cuidado integral da saúde mental da mãe visando preservar um futuro para o binômio (PINTO TM, et al., 2017; SILVA MJS, et al., 2020).

O cuidado a estas pacientes deve ser diferenciado, não restringindo-se apenas a ouvi-las, mas também garantindo que suas dúvidas, medos e preocupações sejam esclarecidas. Fazê-las compreender os próximos passos a seguir, tanto de luto quanto de assistência em saúde da mulher, fazendo sentir não mais como uma parturiente e nem puérpera, mas uma mulher em processo de luto e que precisará de apoio e fortalecimento em sua autoestima e identidade pessoal (MIRANDA CLM, 2011).

Uma das estratégias para minimizar os impactos à saúde mental da mulher com óbito fetal é a implantação da assistência psicológica. No momento vivenciado pelas acadêmicas, uma dificuldade percebida quanto a uma prestação de assistência de qualidade a alguém diante dessa situação foi justamente o problema estrutural. Levando em consideração que isso reflete de forma negativa na saúde mental da mãe, faz-se necessário que os profissionais e gestores reflitam a respeito de tal obstáculo de forma a tentar minimizar ou até excluir essa dificuldade vivida nos hospitais de prestação a assistência à mulher em período gestacional, parto e pós-parto espalhados por todo o Brasil.

No que tange aos problemas estruturais, Santos CS, et al. (2012) demostra também a deficiência estrutural encontrada na maternidade de seu estudo, já que não possuía uma enfermaria específica para acolher mulheres com diagnóstico de óbito fetal e a assistência pós-parto. A carência de espaço físico além de dificultar a assistência, acabava provocando um desequilíbrio emocional muito maior nessa mulher pelo fato de as mães já possuírem um diagnóstico de óbito fetal e ainda serem obrigadas a presenciar outras mulheres com seus filhos, durante esse momento que é tão delicado para elas.

A experiência adquirida durante o estágio supervisionado reforça a ideia de que a assistência prestada à mulheres com óbito fetal deve ser um continuo processo de educação em saúde, tanto dentro das universidades quanto nos setores de trabalho dos profissionais de saúde, a fim de potencializar a construção de novas práticas que estimulem a empatia necessária para a escuta qualificada, que se faz essencial nos cuidados à estas mulheres. 


\section{REFERÊNCIAS}

1. ARAÚJO JL, et al. Sistema Único de Saúde e democracia: a enfermagem no contexto de crise. Revista Brasileira de Enfermagem, 2018; 71(4): 2187-92.

2. BRASIL. Política nacional de atenção integral à saúde da mulher: princícios e diretrizes/ Ministério da Saúde, Secretaria de Atenção à Saúde, Departamento de Ações Programáticas Estratégicas.Brasília: Ministério da Saúde, 2004

3. BRASIL. Ministério da Saúde. Secretaria de Atenção à Saúde. Núcleo Técnico da Política Nacional de Humanização. HumanizaSUS: Documento base para gestores e trabalhadores do SUS/ Ministério da Saúde, Secretaria de Atenção à Saúde, Núcleo Técnico da Política Nacional de Humanização-4. ed. 4. reimp.Brasília: Editora do Ministério da Saúde, 2008.

4. BRASIL. Ministério da Saúde. Secretaria de Atenção à Saúde. Núcleo Técnico da Política Nacional de Humanização. HumanizaSUS: Documento base para gestores e trabalhadores do SUS/ Ministério da Saúde, Secretaria de Atenção à Saúde, Núcleo Técnico da Política Nacional de Humanização-4. ed. 4. reimp. Brasília: Editora do Ministério da Saúde, 2010. 72

5. BRASIL. Ministério da Saúde. Secretaria de Atenção à Saúde. Núcleo Técnico da Política Nacional de Humanização. HumanizaSUS: cadernos de textos: cartilhas da Política Nacional de Humanização. Brasília, DF: Ministério da Saúde, 2010.

6. BRAṠIL. Ministério da Saúde. Secretaria de Atenção à Saúde. Departamento de ações Programáticas Estratégicas. Atenção humanizada ao abortamento: norma técnica/ Ministério da Saúde, Secretaria de Atenção à Saúde. Brasília: Ministério da Saúde, 2011.

7. CARLO WA, TRAVERS CP. Maternal and neonatal mortality: time to act. Jornal de pediatria, 2016; 92(6): 543-545.

8. CARVALHO AJ. Qualidade de vida, ansiedade e depressão em mulheres com aborto espontâneo recorrente: Quality of life, anxiety and depression in women with recurrent spontaneous abortion. Tese (Doutorado em Ciências da Saúde) - Faculdade de Ciências Médicas. Universidade Estadual de Campinas, Campinas, 2019; 107 p.

9. DODOU HD, et al. Sala de parto: condições de trabalho e humanização da assistência. Cadernos saúde coletiva, 2017; 25(3): 332-338.

10. DUARTE MG. Luto na maternidade: construção de cartilha para cuidados em situação de óbito perinatal. Dissertação (Mestrado em Enfermagem) - Faculdade de Medicina de Botucatu. Universidade Esstadual Paulista, Botucatu, 2019; $111 \mathrm{p}$.

11. FARREN J, et al. Posttraumatic stress, anxiety and depression following miscarriage and ectopic pregnancy: a multicenter, prospective, cohort study. American journal of obstetrics and gynecology, 2020; 222(4): 367-367.

12. LANSKY S, et al. Pesquisa Nascer no Brasil: perfil da mortalidade neonatal e avaliação da assistência à gestante e ao recém-nascido. Cadernos de Saúde Pública, 2014; 30: 192-207.

13. LARI LR, et al. Suporte aos pais que vivenciam a perda de um filho neonato: revisão de literatura. Aquichan, 2018; 18(1): 80-94.

14. LEAL MC, et al. Saúde reprodutiva, materna, neonatal e infantil nos 30 anos do Sistema Único de Saúde (SUS). Ciência \& Saúde Coletiva [online]. 2018; 23(6): 1915-1928.

15. LEÓN AR, et al. Depressão e ansiedade na gravidez e a influência na intenção e duração pretendida da amamentação. Dissertação (Mestrado em Medicina) - Faculdade de Medicina. Universidade de Coimbra, Coimbra, $2018 ; 46$ p.

16. LIMA ML, et al. Cuidado humanizado às mulheres em situação de abortamento: uma análise reflexiva. Revista de enfermagem UFPE online, 2017; 11(12): 5074-5078.

17. LOPES BG, et al. A dor de perder um filho no período perinatal: uma revisão integrativa da literatura sobre o luto materno. Revista Stricto Sensu, 2019; 4(2); 29-40.

18. LUNA IJ, MORE CO. Narrativas e processo de reconstrução do significado no luto. Revista M. Estudos sobre a morte, os mortos e o morrer, 2019; 2(3): 152-172.

19. MADEIRO AP, RUFINO AC. Maus-tratos e discriminação na assistência ao aborto provocado: a percepção das mulheres em Teresina, Piauí, Brasil. Ciência \& Saúde Coletiva, 2017; 22(8): 2771-2780.

20. MENDONÇA SA. Interrupção espontânea da gravidez, morte fetal e perda perinatal: luto e fatores protetores. Dissertação (Mestrado em Psicologia) - Faculdade de Psicologia. Universidade de Lisboa, Lisboa, $2018 ; 81$ p.

21. MENEZES RR, et al. A Importância da Amamentação na Formação de Vínculos Afetivos Saudáveis Entre Mamãe/Bebê. BIUS-Boletim Informativo Unimotrisaúde em Sociogerontologia, 2019; 12(5): 1-15.

22. MIRANDA CLM. O sentido do ser-mãe-que-engravidou-após-óbito-fetal: possibilidades assisntenciais de e para a enfermagem / The Feeling Of Being-Mother-That-Got-Pregnant-After-Fetal-Death: Assistencial Possibilities of and for Nursing. Tese (Doutorado em Enfermagem) - Escola de Enfermagem Anna Nery. Universidade Federal do Rio de Janeiro, Rio de Janeiro, 2011, $120 \mathrm{p}$.

23. O'DWYER GO, et al. Avaliação dos serviços hospitalares de emergência do programa QualiSUS. Ciência \& Saúde Coletiva, 2009; 14(5): 1881-1890.

24. PARADA CMGL, CARVALHÃES MBL. Childbirth care: contributing to the debate on human development. Revista LatinoAmericana de Enfermagem, 2007, 15(número especial); 792-798.

25. PASTOR MSM, et al. A experiência da perda perinatal à partir da perspectiva dos profissionais de saúde. Revista Latino-Americana de Enfermagem, 2011; 19(6);1405-1412.

26. PINTO TM, et al. Depressão e ansiedade maternal e crescimento fetal-neonatal. Jornal de Pediatria, 2017; 93(5); 452459

27. POSSATI AB, et al. Humanização do parto: significados e percepções de enfermeiras. Escola Anna Nery, 2017; 21(4): 1-6.

28. SANTOS CS, et al. Percepções de enfermeiras sobre a assistência prestada a mulheres diante do óbito fetal. Escola Anna Nery, 2012;16(2);277-284.

29. SANTOS HFL, ARAUJO MM. Políticas de humanização ao pré-natal e parto: uma revisão de literatura humanization the policies prenatal and childbirth: a literature. Revista científica FACMAIS, 2016; 6(2): 55-64.

30. SILVA L, et al. Percepção das Mulheres em Situação de Abortamento Frente ao Cuidado de enfermagem/ Perception of women in abortion situation front of nursing care/ Percepción de mujeres em abortación com cuidado de enfermeira. Revista Ciência Plural, 2020; 6(1): 44-55.

31. SILVA MJS, et al. Depressão na gravidez. SMAD Revista Eletrônica Saúde Mental Álcool e Drogas, $2020 ; 16(1) ; 1$ 12.

32. SOARES MCS, et al. Práticas de enfermagem na atenção às mulheres em situação de abortamento. Revista da Rede de Enfermagem do Nordeste, 2012; 13(1): 140-146. 